\title{
Rail Noise Levels Around Dammam Railway Track and Reported Impacts on Human Health
}

\section{Uneb Gazder}

Department of Civil Engineering, University of Bahrain, Isa Town, Bahrain

\section{Abstract}

Noise pollution has become a major concern due to its effects on human health. Therefore, the author has chosen to study the effects of noise pollution caused by trains in the rural areas near Dammam city of Saudi Arabia in this study. An attempt is made to monitor the railway noise pollution at certain distances from the railway track. $T$-test was applied to investigate statistical significance of the difference in noise levels at these distances at different timings. Moreover, a survey was conducted among people, who were exposed to noise levels at different distances from the track,

Corresponding Author: Uneb Gazder unebgazdar@gmail.com Received: 18 September 2018 Accepted: 10 October 2018 Published: 15 October 2018

Publishing services provided by Knowledge $\mathrm{E}$

(c) Uneb Gazder. This article is distributed under the terms of the Creative Commons

Attribution License, which permits unrestricted use and redistribution provided that the original author and source are credited.

Selection and Peer-review under the responsibility of the Sustainability and Resilience Conference Committee. to have their opinions about the noise level and its effects. It was found that noise levels diminish significantly at $20 \mathrm{~m}$ from the railway track. But the noise levels were still above the recommended Saudi standards for rural areas (which was the type of study area) at this distance. The noise levels were found within the recommended limits after $50 \mathrm{~m}$ from the railway track in the day time. After which it started to increase again that could be attributed to the presence of highways generating traffic noise. The noise levels were more than recommended for all locations during night time. Moreover, it was found that residential and educational land uses were allowed within $50 \mathrm{~m}$ from the track. This could result in continuous exposure and sustained effects on inhabitants' health. The most common problems due to railway noise were reported to be annoyance, sleep disturbance and nervous turbulence. Sleep disturbance and nervous turbulence were reported by the people who live within $50 \mathrm{~m}$ from the track, where the noise levels were found to be above the allowable standards. It was recommended by the survey respondents to either construct a barrier around the tracks or relocate them.

Keywords: Dammam, Rural Areas, Rail Noise, Health Effects

\section{Introduction}

There has been substantial development in all sectors of life including communication G OPEN ACCESS 
bad impact on the society in the form of pollution. Engineers and developers are working to mitigate and reduce this impact in order to maintain life quality and health. Transportation, including road, railways, air and sea, is considered to be one of the highest contributor to pollution along with industries and power production (Smith, 2011).

One of the crucial aspects of pollution that has resulted from development and innovations is noise pollution. Noise pollution takes place when there is either excessive amount of noise or unpleasant sound that causes temporary disruption in the natural balance (MED-EL, 2015). It has become very difficult or even impossible to escape noise pollution in our daily activities. It can be sourced by inside or outside homes. Many of the electrical appliances, such as air-conditioning, inside home emit undesired noise. Health and mood of people can have unnoticeable effects by these home appliances. On the other hand, massive noise coming out of heavy machines and factories might cause chronic health problems, such as hearing and headache problems, to the people around them. The effects of noise pollution are hidden as it is not a sudden disordering but can have severe effects on health with continuous exposure. Researchers have found that exposure to traffic noise can increase the risk of suffering from heart attack. World Health Organisation (WHO) estimates that the region of east Europe has at least one million disability adjusted life years lost due to diseases induced by the noise (Young and Woods, 1970).

Many cities, including those of third world countries, are relying on railway transport for goods and passenger transportation. This mode of transport has been promoted as a solution for congestion on the highways and consequently reduces the emissions due to traffic. However, its widespread use has other impacts on the communities including noise pollution. Railway noise is considered as be a serious issue when tracks/stations are placed near or inside the city centres where its noise combines with other sources. Therefore, community resistance has been observed against railway noise in some countries including China (He et al., 2015).

The major noise pollution in GCC and precisely in Saudi is caused by cars due to the high vehicular demand resulting in congestion. However, there are some places close to the railway tracks and stations where high levels of noise, sourced by the train engines and track friction, are experienced. These locations mainly include some villages, and this could be the reason that we could not find any studies in which noise levels for these locations are studied. Researchers often focus on densely populated urban areas for studying environmental impacts of transportation. 
This study had the following objectives. Firstly, reviewing railway noise and its technical measurements and effects. Secondly, measuring noise pollution on certain points surrounding Dammam's railway tracks. Third objective was determining perception, related to effects of noise on health, of the population exposed to this noise. Lastly, analysing the results to recommend practical and technical solution for mitigation of the noise.

There are some limitations that were faced throughout this study. Firstly, there is lack of available information about Dammam's railway and train system. Secondly, the readings and noise measurements were taken by normal phone app, instead of a proper measuring device which would have been more accurate but costly as well.

\section{Noise Pollution}

While noise is a form of sound level, but there is a difference between the practical meanings of these two terms. Sound can be defined as mechanical wave that results from the back and forth vibration of particles that travel in the medium (Smith, 2014). Noise can be defined as unwanted sound judged to be unpleasant, loud or disruptive to hearing. From a scientific standpoint, noise is indistinguishable from sound, as both are vibrations through a medium, such as air or water. The difference arises when the brain receives and perceives a sound. In experimental sciences, noise can refer to any random fluctuations of data that hinders perception of an expected signal (Bertland, 1970). There are various causes of noise including, vehicles, music instruments, sirens/alarms and industrial tools and equipment (de Bell, 1970).

Generally, the noise can be understood and felt as an annoyance to hearing sourced by different items in the daily activities. It is measured in a unit called the decibel $(\mathrm{dBA})$. Decibel is a unit to measure the intensity or the level of sound and its measured by comparing to a logarithmic scale assigned by scientists to determine the levels (Galloway et al., 1969).

\subsection{Noise thresholds}

The permitted noise level is 125 decibels as per the Environment Protection Rules 1999. It can be sourced from important uses and non-important uses. Important uses include transportation vehicles, such as; cars, trains and aircraft. Noise from heavy traffic can reach $85 \mathrm{~dB}$ which is the level having potential to cause hearing problems with time (Harmsens, 2017). The threshold for hearing is from above Zero to 5 which is barely to 
be heard. Range between 20 and 40 is referred to as a quiet place like home at nights. The noise levels can be easily detected when it reaches $65 \mathrm{~dB}$, for e.9., $75 \mathrm{~dB}$ is the case where there is highway traffic with heavy vehicles (Carlon, 2015).

\subsection{Noise types}

Noise varies in its levels and types which also varies the risks associated with it. Below are the different types of the noise that affects everyone on daily bases (Thomson, 2017).

\subsubsection{Intermittent noise}

This type of noise is too frequent and it increases and decreases quickly (Christina, 2011).

\subsubsection{Continuous noise}

This type of noise is emitted continuously without interruption for a certain while. Usually it comes from a running machinery, ventilation or ACs, etc. It can be measured by sound level meter by exposing it to the noise for few minutes (Stansfeld and Matheson, 2003).

\subsubsection{Impulsive noise}

This noise is associated mainly with construction fields and explosive processes. It is a result of a sudden substantial increase in sound like a bang; a sudden burst of noise having fast and surprising nature. It can be measured with a meter called personal noise dosimeter or the normal sound level meter (Oyedepo and Saadu, 2009).

\subsubsection{Low frequency noise}

Low recurrence noise makes up some portion of the texture of our everyday soundscape, such as; low foundation murmuring from power plants. This is additionally the hardest type of noise to decrease from the source, so it can simply spread around longer distances. For low recurrence noise, you ought to utilize a sound level meter with Third Octave Band (Waye et al., 2001). 


\subsection{Rail noise pollution}

According to Europal, Rail noise is a sound emission sourced by the operation of trains, trams and metro (Carlon, 2015). There are a wide variety of sources and causes of this type of noise like locomotive acceleration, braking, rail curving squeal sound and vibration from rail corrugation. Rail noise starts with train engines with different types of engines producing varying the levels of noise, and then with the movement of rail, noise is sourced by wheels turning or rolling. Furthermore, horns, bells and whistles are also major types of the noise during acceleration of trains. Rail noise has been under focus due to the reaction of people against it (Bistrup et al., 2001). Consequently, many rules and regulations were set to evaluate the amount of noise and to avoid their effects on health and comfort (van Haaren and Verheijen, 1999).

Rolling noise is considered to be the major source of railway noise and it can be briefly defined as the noise which is resulted from absolute friction between the wheels and the track. It is caused by the effect of amplitude undulation of the wheel and the rail track. This type of noise can be reduced through reduction in vibration in wheel, sleepers and rail track by adding a damping treatment, reducing the noise by barriers, employing smaller wheels and rails and using proper disk brakes instead of ordinary iron blocks (Ritchard, 2011).

Curve squeal is high sound tone which occurs usually at the curves when wheels of the rail are turning on tight radius (Young and Woods, 1970). Typically, squeal can be distinguished by its higher level of noise in comparison with wheels rolling and it adds 20dBA more in level of noise (Millward, 2011). It can be controlled by reducing the friction between the rail wheels and rail tracks through lubricants (de Bell, 1970). Poor rail profile can also be a big cause of squeal noise by generating more stress on the wheels at curves, thus, selecting better properties as well as ongoing maintenance can assure more durable system with less effect of the stresses and noises.

\subsection{Noise barriers}

Noise barriers have been considered as a basic solution to reduce exposure to rail noise. They are designed exterior structures made of different sound proof properties in order to protect the inhabitants and sensitive land use areas from the noise (Alen, 2014). There are three different types of noise barriers which may be applied, namely: noise berms, noise walls and the combination of noise berms \& walls. 


\section{Methodology}

Saudi Railway Organization (SRO) has a total railway track length of $1775 \mathrm{Km}$. The major railway line starts in Dammam airport and in eastern part of Saudi Arabia and goes till Riyadh, the capital of Saudi Arabia, situated in middle of the country. The major lines connect some industrial areas, farms and military areas to ports and villages. SRO has 3 major lines:

1. Passenger's line: Railway number 1 which is double line and total of $733 \mathrm{Km}$ linking Riyadh to Dammam passing through Hoffof and Bugaig.

2. Cargo line: Railway number 2 which is for transporting the materials and equipment only. It is $556 \mathrm{Km}$ and links Abdulaziz port in Dammam to Riyadh dry port.

3. Minor lines: They are $486 \mathrm{Km}$ that link locations of industrials areas and some military areas.

\subsection{Noise level observations}

We started by measuring the noise level of the train over several distances; first interval was closest to the railway track which is located within 20 meters. The second one was from the nearest village which is 20-50 meters away. Third interval was at 50-100 meters away and last point was at 130 meters away from the tracks. At each interval 12 readings were recorded and at 2 times a day (evening and morning). The measurements of noise were taken by an iPhone application called (Decibel 10) which is created for such sound measurements. The measurements of noise through mobile applications has been proved to give satisfactory results (Kardous and Shaw, 2014). At each selected location, the application was operated for a continuous measuring period of 5-10 min during which twelve readings of noise were automatically recorded and saved.

\subsection{Questionnaire survey}

Second step was conducting a questionnaire survey to know respondents' opinion about effects of noise on them from health perspectives. The questionnaire was set consisting of 9 questions. A brief explanation of the questions is given below.

First Question was about the distance between the respondent's place of exposure and railway track. Question two was about the type of activity which that person is 
involved in when he/she is near the railway track. Question 3 and 4 were about gender and age. Question 5,6 \& 7 have been included to know the perspective related to noise level and its effects on them. Question 8 was to ask the respondent about how the noise level affects their daily activities. Last question was to suggest probable solutions for the railway noise by selecting reasonable options from the given list.

\subsection{Analysis methods}

The noise observations were compared to get an understanding of noise at different distance intervals. Paired t-test was done in the analysis to find whether there is significance difference between the noise levels at different distances from the track, and different timings. Paired t-test is based on the differences between the values of each pair. The paired t-value can be calculated using equations 1 and 2 (Ritchard, 2017).

$$
\begin{gathered}
\mathrm{SE}(\mathrm{d})=\sqrt{\frac{s d}{n}} \\
T=d \backslash S E(d)
\end{gathered}
$$

Where sd represents the standard deviation for the difference, $n$ is the number of pairs in the data and $d$ is the average difference between the pairs. T-value for the difference in noise datasets was compared with a standard t-value at $95 \%$ confidence having $n-1$ degrees of freedom. If the t-value was higher than standard $t$-value then the difference between the datasets is significantly higher and vice versa (Ritchard, 2017).

\section{Data Analysis}

Average of noise observations, taken at different distances from the railway track at two different timings is compared in Figure 1. All these averages were compared using the paired t-test (as described in sub-section 3.3) for all possible pairs of data. The $\mathrm{t}$-value for all the tests were higher than the standard t-value hence, it can be said that the average noise levels varies from one location to another location as well as between the observation times.

It should be noted at this point that 12-1AM was selected as more trains are scheduled to pass on the tracks at these times. Whereas, lesser number of trains are scheduled between 1-2PM. Therefore, higher noise levels are observed during 12-1AM at all the locations except at $130 \mathrm{~m}$ away from the track. It can also be observed that the noise 


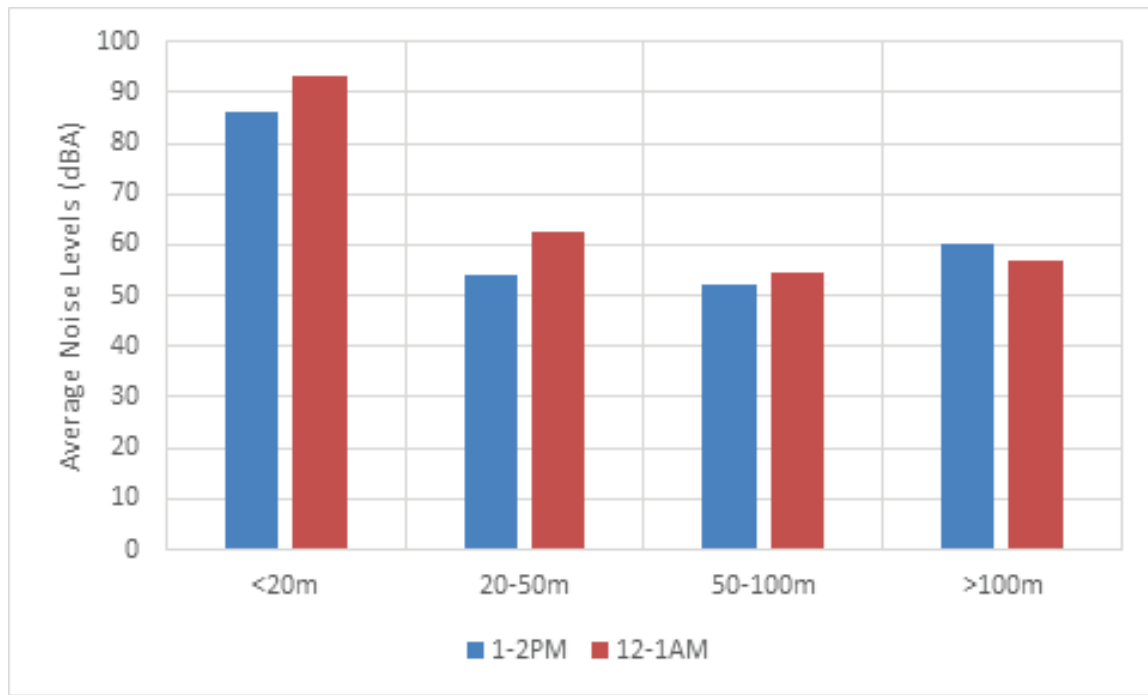

Figure 1: Average Noise Levels at Different Locations and Timings.

levels at this location increased although they were decreasing for observations with increase in distance for other intervals. It was observed during the visit of observation sites that roads are constructed at $100 \mathrm{~m}$ away from the track. So, the peculiar noise levels at 130m, which are not consistent with the trends shown by rest of the data, can be attributed to road traffic noise.

According to Saudi environmental standards, community noise should be in the range of 55-50 dBA during day times and within 45-50 during night times (Zytoon, 2016). The night-time ranges are violated at all the study locations, mainly because of the movement of trains. The day-time standards are maintained at $20 \mathrm{~m}$ and $50 \mathrm{~m}$ away from the track.

\subsection{Survey data description}

General characteristics about the survey respondents are given in table 1. Majority of the survey respondents belonged to a mature age group of above 20 years. Approximately equal number of responses were taken at different distances intervals from the railway track. Majority of the respondents were male due to cultural values in Saudi Arabia wherein interviewing women, by male data collectors of this study, is comparatively difficult. There was a significant number of respondents who reported living or studying within 10om from the railway track which means continuous exposure.

Interestingly, people did not rate the noise level very high and majority of them reported that noise level did not affect their health or efficiency. The possible reasons for this trend could be that due to long exposure to the rail noise (as majority of them 
TABLE 1: General Characteristics of Respondents.

\begin{tabular}{l|c|}
\hline $\begin{array}{l}\text { Variable } \\
\text { Total respondents }\end{array}$ & Data \\
\hline Number of people exposed within 20m & 26 \\
\hline $\begin{array}{l}\text { Number of people exposed within 20-50m } \\
\text { Number of people exposed within 50-10om }\end{array}$ & 21 \\
\hline Number of people exposed after 10om & 23 \\
\hline$\%$ of people living near track & 27 \\
\hline$\%$ of people doing business near track & 50 \\
\hline$\%$ of people passing near the track & 15 \\
\hline$\%$ of people studying near the track & 30 \\
\hline$\%$ male respondents & 5 \\
\hline$\%$ of respondents having age between 15-20 years & 67 \\
\hline$\%$ of respondents having age between 20-40 years & 25 \\
\hline$\%$ of respondents having age between $40-60$ years & 45 \\
\hline$\%$ of respondents having age above years & 25 \\
\hline$\%$ of respondents thinking they are affected by noise & 5 \\
\hline $\begin{array}{l}\text { Average rail noise ranking by the respondents (1=low, } \\
\text { 5=high) }\end{array}$ & 32 \\
\hline $\begin{array}{l}\text { rail noise } \\
\text { rail noise }\end{array}$ & 3.2 \\
\hline work/study due to rail noise & 30 \\
\hline
\end{tabular}

are living/working/studying near the track), respondents are used to it. The respondents which reported having effects of noise on them reported sleep disturbance as the main health issue associated with exposure to rail noise. It has already been established that noise levels exceed the Saudi environmental standards for night time at locations. More than $40 \%$ of the respondents suggested to relocate the railway track from the current location and/or construct noise barriers around them.

\subsection{Locational comparison}

The perceptions of respondents, exposed to rail noise at different distances, were compared and the results are shown in figures $2-4$. It can be observed that more than $40 \%$ of the respondents were living or working close to the railway track (less than $50 \mathrm{~m}$ away). Furthermore, there are approximately $20 \%$ of respondents who are studying in the same proximity. 


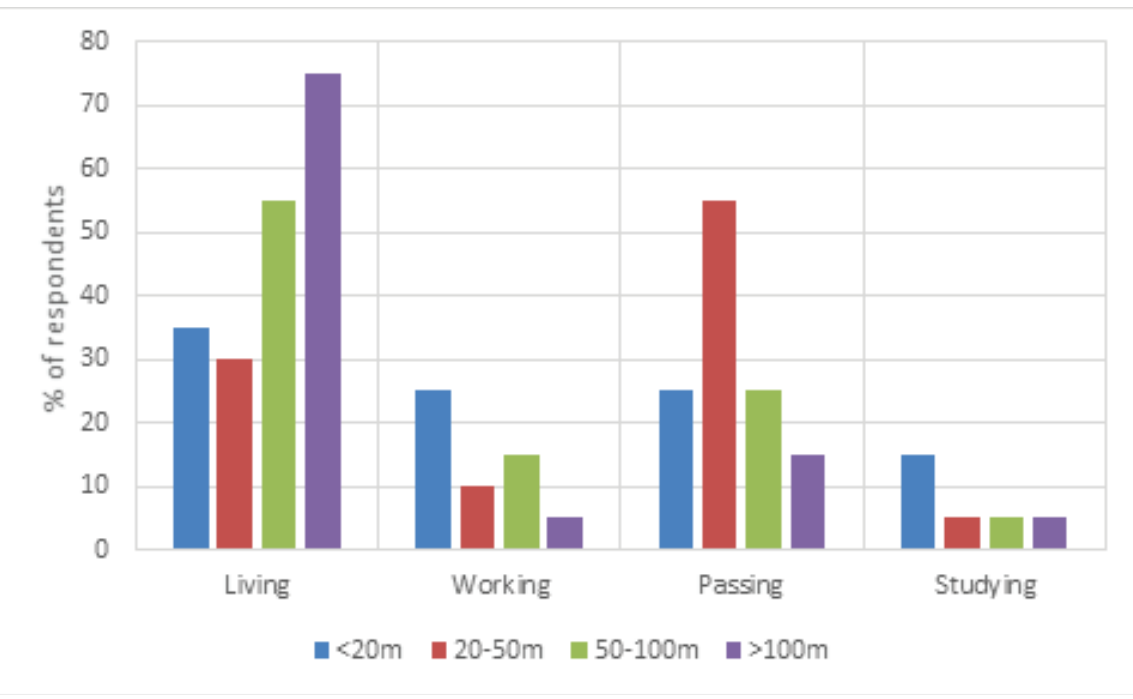

Figure 2: Comparison of Respondents' Activities.

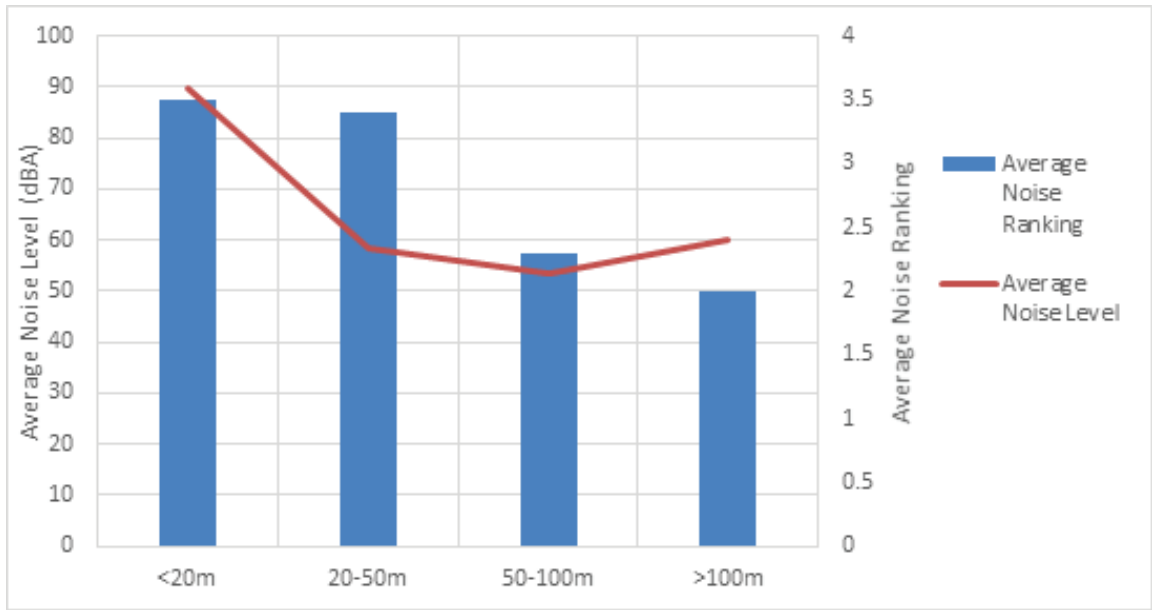

Figure 3: Comparison of Noise Levels and Rankings.

The ranking of the noise level, according to respondent's perception about rail noise, decreases with the increase in distance from track (see Figure 3). However, a considerable decrease is shown in the ranking by respondents who are 5om away, while the noise level reduces considerably after $20 \mathrm{~m}$. Moreover, the noise level at more than 10om from the track is slightly higher, than previous interval, due to highway traffic but the noise ranking keeps on getting lower at this interval. From these observations, it could be said that people's perception about environmental hazards (such as noise) can differ slightly from the actual data.

Nervous turbulence was mainly reported by respondents who are exposed at less than $20 \mathrm{~m}$ from the track (see Figure 4). Sleep disturbance was a common phenomenon which is reported by most of the respondents, irrespective of their distance from the track. It should also be noted that Saudi environmental standards for night times (50 


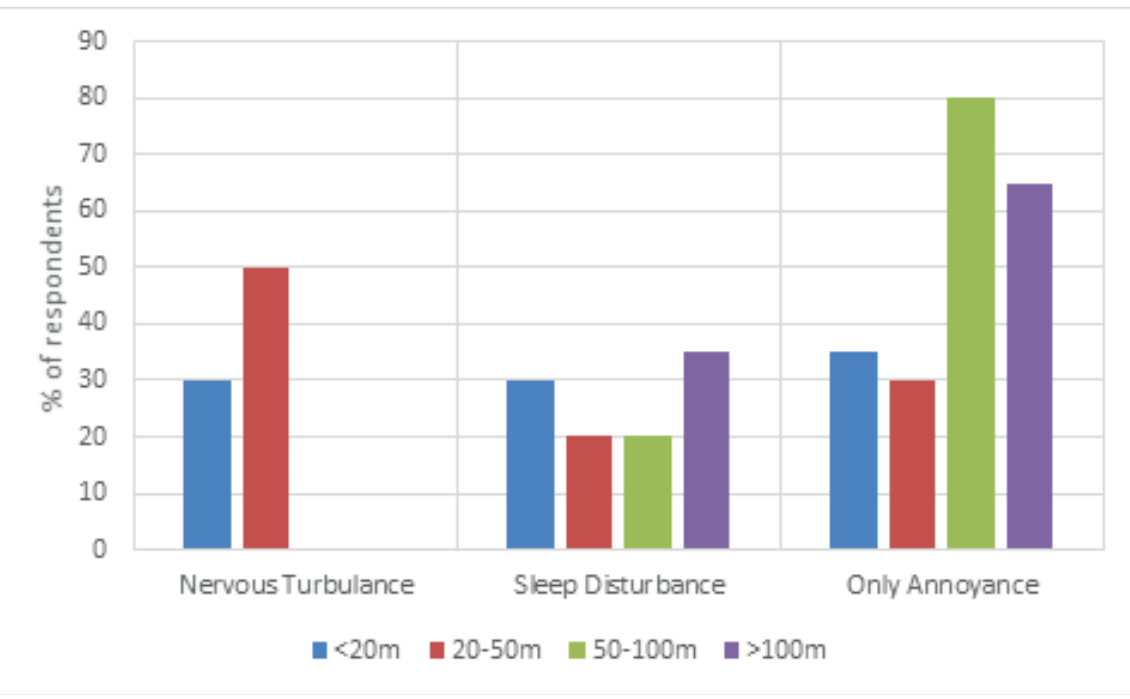

Figure 4: Comparison of Perceived Health Problems.

$\mathrm{dBA}$ ) have been violated at all these locations (Zytoon, 2016). This data shows that violating these standards is causing nervous turbulence (within $50 \mathrm{~m}$ from the track for day time) and sleep disturbance to the exposed population. Annoyance was reported by majority of the respondents who are exposed at more than $50 \mathrm{~m}$ away from the track, while it is reported by some respondents at other intervals as well.

\section{Conclusions and Recommendations}

The aim of the study was to investigate the railway noise pollution and the perception of people about it. It was found that noise levels have significantly reduce when the distance from the track is increased by $20 \mathrm{~m}$. The frequency/schedule of trains also has a significant impact on the noise levels.

We found that most of the survey respondents are used to high noise levels due to consistent exposure, therefore, do not believe that noise pollution affects them. Although, rail noise is more than allowable Saudi environmental standards on most of the surveyed locations, but there was a significant population of respondents who live/work/study near the track (within 50m). Nervous turbulence and sleep disturbance have been reported as the main health issues, related to the exposure of rail noise, on locations where it exceeds the environmental standards. Majority of the respondents suggested construction of noise barriers or relocation of railway tracks, away from population, to reduce the impacts of their noise on them. The relocation of residential areas away from the track would also be relevant in this case. Other possible solution for reducing noise could be maintenance and updating of railway 
system to comply with modern European standards which are considered a benchmark for modern railway systems.

\section{Author's Biography}

Dr. Uneb Gazder is an Assistant Professor in Department of Civil Engineering at University of Bahrain. He completed his PhD, with specialization in Transportation Planning, from King Fahd University of Petroleum and Minerals in 2014. His areas of research include: Artificial Neural Networks, Traffic Forecasting, Mode Choice Modeling and Environmental Impacts of Transportation.

\section{References}

[1] Bertland, T. (1970). Noise: The third pollution. Public Affairs Committee, Inc.

[2] Bistrup, M. L., Hygge, S., Keiding, L., Passchier-Vermeer, W. (2001). Health effects of noise on children and perception of the risk of noise. Copenhagen: National Institute of Public Health.

[3] Carlon, S. (2015). What are the four different types of noise. Available: http:// www.cirrusresearch.co.uk/blog/2015/01/4-different-types-noise/. (Accessed on 22th April 2017).

[4] Christina, S. (2011). Noise research in special supplement. (Online) Available: https: //www.cdc.gov/niosh/updates/upd-02-03-11.htm. (Accessed $15^{\text {th }}$ June 2017).

[5] de Bell, G. (1970). A future that makes ecological sense. The environmental handbook. New York: Ballantine Books.

[6] van Haaren, E., Verheijen, E. (1999). A literature study and a proposal for a measurement protocol for curve squeal noise, Dutch title: Literatuurstudie en een voorstelMeetprotocol Booggeluid. NSTO-report NSTO/99/8110026/038.

[7] Kardous, C. A., Shaw, P. B. (2014). Evaluation of smartphone sound measurement applications. The Journal of the Acoustical Society of America, 135(4), EL186-EL192.

[8] Harmsens, N. (2017). Horn noise. ABC news, Available: http://www.abc.net. au/news/2015-02-19/residents-complain-about-train-horns-noise/6152474. (Accessed on 18th April 2017).

[9] He, G., Mol, A. P., Zhang, L., \& Lu, Y. (2015). Environmental risks of highspeed railway in China: public participation, perception and trust. Environmental Development, 14, 37-52. 
[10] MED-EL. (2015). Sound and Noise. Available: https://blog.medel.com/what-issound-what-is-noise/. (Accessed on 15th February 2017).

[11] Millward, D. (2011). Road and rail transport. The Telegraph, Available: http://www.telegraph.co.uk/news/uknews/road-and-rail-transport/8340584/ Number-of-homes-to-be-hit-by-high-speed-train-noise-cut.html. (Accessed on 12th January 2017).

[12] Oyedepo, O. S., \& Saadu, A. A. (2009). A comparative study of noise pollution levels in some selected areas in Ilorin Metropolis, Nigeria. Environmental Monitoring and Assessment, 158(1-4), 155.

[13] Ritchard, T. (2017). Paired t test, Available at: http://math.tutorvista.com/statistics/ paired-t-test.html (Accessed on 15th Nov 2017).

[14] Ritchard, T. (2011). Noise. Available: http://whatis.techtarget.com/definition/noise. (Accessed on18th Feb 2017).

[15] Smith, A. (2016). Sound wave. Available: http://www.physicsclassroom.com/ Class/sound/u11lic.html. (Accessed on 12th March 2017.

[16] Smith, P. (2014). The world is too loud. Available: (4) https://psibrone.wordpress. com/2014/06/09/the-world-is-too-loud-2/. (Accessed on 20th Sep 2017).

[17] Stansfeld, S. A., \& Matheson, M. P. (2003). Noise pollution: non-auditory effects on health. British Medical Bulletin, 68(1), 243-257.

[18] Thompson, D. (2008). Railway noise and vibration: mechanisms, modelling and means of control. Elsevier, Amsterdam, The Netherlands.

[19] Waye, K. P., Bengtsson, J., Kjellberg, A., Benton, S. (2001). Low frequency noise "pollution" interferes with performance. Noise and Health, 4(13), 33.

[20] Young, M. F. Woods, D. L. (1970). Threshold noise levels. Texas Transportation Institute, December 1970, Accession Number: 00222602.

[21] Zytoon, M. A. (2016). Opportunities for environmental noise mapping in Saudi Arabia: A case of traffic noise annoyance in an urban area in Jeddah city. International Journal of Environmental Research and Public Health, 13(5), 496. 